\title{
The Role of GERD Questionnaire (GERD-Q) for Reflux Esophagitis
}

\author{
Kaka Renaldi \\ Division of Gastroenterology, Department of Internal Medicine, Faculty of Medicine \\ Universitas Indonesia/Dr. Cipto Mangunkusumo General National Hospital, Jakarta
}

Gastroesophageal reflux disease (GERD) is a disease commonly encountered in daily clinical practice worldwide. ${ }^{1}$ When the reflux has caused esophageal mucosal damage, it is classified as GERD with reflux esophagitis (RE). ${ }^{2}$ There is no complete epidemiological data on this disease in Indonesia. Still, a study from Cipto Mangunkusumo hospital by Syam et al reported a significant increase of esophagitis incidence, an average of $13.13 \%$ per year. ${ }^{3}$ Untreated GERD negatively affects the patients' quality of life, but RE also increases Barret's Esophagus and esophageal cancer risk. ${ }^{4}$ Thus, early recognition to prevent GERD progression or complications is crucial.

Current national guidelines recommend the symptomsbased approach using the GERD questionnaire (GERDQ) with a cut-off score of 8 or more to diagnose GERD and measure the response to therapy. ${ }^{5,6}$ The GERDQ has been translated into the Indonesian language and validated by Simandibrata et al. in 2017..$^{7,8}$ In the presence of alarming symptoms and patients not responding to the empirical PPI treatment, upper gastrointestinal endoscopy (UGIE) is conducted, which is also the gold standard for diagnosing RE and determining its severity according to the Los Angeles (LA) classification system. ${ }^{5,10}$ Recently, several studies have reported the potential clinical value of GERDQ in diagnosing RE.

The largest study involving 8,065 patients comes from China, conducted by Bai et al in 2013. The study reported that the probability of Chinese patients with reflux esophagitis finding at UGIE rise with the increase of the GERDQ score. In the study, 29.1\% of patients with a GERDQ score of 8-10 had RE, while in patients with a GERDQ score of $11-18,35.9 \%$ had RE. ${ }^{11}$ Recent studies by Wang et al in 2017 and Albayati et al in 2019 also reported that the GERDQ score is positively correlated with esophagitis LA severity, $(\mathrm{r}=0.244, \mathrm{p}<0.01)$ and $(\mathrm{r}=0.99, \mathrm{p}<0.01)$, respectively. ${ }^{12,13}$ Currently, two similar studies have also been conducted in Indonesia.
A study by Siahaan et al in 2020 reported that the risk of RE was 2.6 times greater in patients with a GERDQ score of eight or more than in patients with a GERDQ score $<8, \mathrm{p}=0.012 .{ }^{14}$ This result is similar to the previous study by Simarta et al. in 2019, which reported that the GERDQ score was strongly correlated with esophagitis finding at endoscopy, $r$ $=0.643$ with $\mathrm{p}<0.001 .{ }^{13}$ Both studies showed an increasing trend of advancing esophagitis severity with increasing GERDQ score. However, as both studies were underpowered due to the small sample size, this trend was not statistically significant. ${ }^{13,14}$ Still, the possibility of using GERDQ to diagnose esophagitis and distinguish its grading clinically is promising.

First, the questionnaire provides a simple, convenient, and noninvasive examination that can also be completed through telemedicine, limiting face-toface patient contact during the COVID-19 pandemic. Second, in all studies previously mentioned, $70-90 \%$ of RE patients were suffering from Grade A and Grade B, which were clinically classified as mild esophagitis and mostly treated pharmaceutically., ${ }^{5,1-14}$ Thus, patients with a moderate or severe GERDQ score may be safely diagnosed and aggressively managed without further examination, especially in healthcare facilities where endoscopy services are not readily available. However, there are still cases where endoscopy examination cannot be avoided.

Typical symptom evaluation, such as heartburn and regurgitation, is key to the GERDQ diagnosis, but atypical GERD symptoms may also occur, with or without the cardinal symptoms, especially in Asia. ${ }^{15}$ Bai et al reported that one-third of 1,435 endoscopic RE patients did not have either heartburn or regurgitation and had a low GERDQ score. Therefore, a low GERDQ score cannot exclude the probability of patients suffering from RE. ${ }^{11}$

Another issue in using GERDQ over endoscopy is that a high GERDQ score cannot totally eliminate the chance of upper GI cancer. Out of the 8,065 patients 
in the Bai et al study, 28 patients were found to have malignancies. All patients presented without alarm features, and $35.7 \%$ of these patients had a GERDQ score $>8$, which suggests without upper endoscopy, this patient's group may only be diagnosed and treated as GERD. ${ }^{11}$

In conclusion, GERDQ held promise in guiding the management of GERD with reflux esophagitis. Further work is encouraged to prove its clinical value in diagnosing esophagitis and estimating its severity for Indonesian patients. However, endoscopy examination should not be forgotten, especially in evaluating patients with atypical GERD symptoms and malignancies screening in high-risk patients.

\section{REFERENCES}

1. El-Serag HB, Sweet S, Winchester CC. Update on the epidemiology of gastro- oesophageal reflux disease: a systematic review. Gut 2014;63:871-80.

2. Fock KM, Talley NJ, Fass R, Goh KL, Katelaris P, Hunt $\mathrm{R}$, et al. Asia-Pacific consensus on the management of gastroesophageal reflux disease: update. J Gastroenterol Hepatol 2008;23:8-22.

3. Syam AF, Abdullah M, Rani AA. Prevalence of reflux esophagitis, Barret's esophagus and esophageal cancer in Indonesian people evaluation by endoscopy. Canc Res Treat 2003;5:83.

4. Ronkainen J, Talley NJ, Storskrubb T, Johansson SE, Lind T, Vieth M, et al. Erosive esophagitis is a risk factor for Barrett's esophagus: a community-based endoscopic follow-up study. Am J Gastroenterol 2011;106:1946-52.

5. The Indonesian Society of Gastroenterology. National consensus on the management of gastroesophangeal reflux disease in Indonesia. Acta Med Indones 2014;46:263-71.

6. Jones R, Junghard O, Dent J, Vakil N, Halling K, Wernersson B, et al. Development of the GerdQ, a tool for the diagnosis and management of gastro-oesophageal reflux disease in primary care. Aliment Pharmacol Ther 2009;30:1030-8.

7. Simadibrata M, Rani A, Adi P, Djumhana A, Abdullah M. The gastro-esophageal reflux disease questionnaire using Indonesian language: a language validation survey. Med J Indones 2011;20:125-30.

8. Siahaan LA, Syam AF, Simadibrata M, Setiati S. The validity and reliability test of the Indonesian version of GERD-QOL questionnaire. Acta Med Indones 2017;49:3-9.

9. Lundell LR, Dent J, Bennett JR, Blum AL, Armstrong D, Galmiche JP, et al. Endoscopic assessment of oesophagitis: clinical and functional correlates and further validation of the Los Angeles classification. Gut 1999;45:172-80.

10. Bai Y, Du Y, Zou D, Jin Z, Zhan X, Li ZS, et al. Gastroesophangeal reflux disease questionnaire (GerdQ) in real-world practice: a national multicenter survey on 8065 patients. Journal of Gastroenterology and Hepatology Foundation. 2013;28:626-31.

11. Wang M, Zhang JZ, Kang XJ, Li L, Huang XL, Aihemaijiang $\mathrm{K}$, et al. Relevance between GerdQ score and the severity of reflux esophagitis in uygur and han chinese. Oncotarget 2017:8:74371-7.

12. Albayati S, Khalaf FD. Gastroesophangeal reflux disease questionnaire score and endoscopic findings in patients with gastroesophangeal reflux disease. Mustansiriya Medical Journal 2019;18:63-9

13. Simarta DO, Wahyudi Y, Bestari MB, Supriadi R. Relationship between gastroesophangeal reflux disease questionnaire (GERD-Q) score and reflux esophagitis in gastroesophangeal reflux disease (GERD) suspected patients in Bandung. Indones J Gastroenterol Hepatol Dig Endosc 2019;20:154-60.

14. Siahaan B, Arles, Azhari W. Relationship between GERD-Q score with esophagitis findings in endoscopy. The Indonesian Journal of Gastroenterology, Hepatology, and Digestive Endoscopy. 2020; 21:176-80.

15. Hunt R, Armstrong D, Katelaris P, Afihene M, Bane A, Bhatia $\mathrm{S}$, et al. Global perspective on gastroesophangeal reflux disease. WGO Global guidelines 2015 